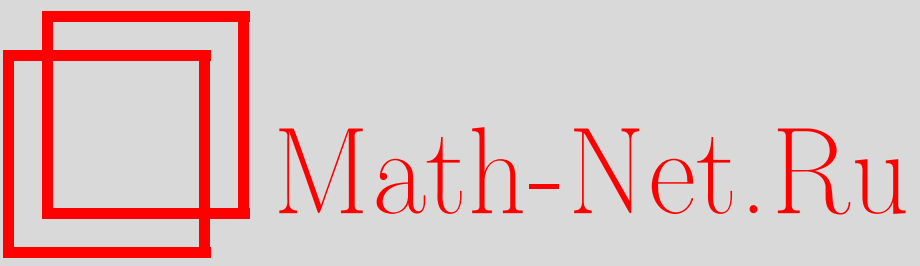

И. А. Баталин, И. В. Тютин, БРСТ-инвариантная алгебра связей в терминах коммутаторов и квантовых антискобок, ТМФ, 2004, том 138, номер 1, 3-22

DOI: https://doi.org/10.4213/tmf4

Использование Общероссийского математического портала Math-Net.Ru подразумевает, что вы прочитали и согласны с пользовательским соглашением

http://www.mathnet.ru/rus/agreement

Параметры загрузки:

IP: 3.85 .73 .92

26 апреля 2023 г., 16:24:11 
ТЕОРЕТИЧЕСКАЯ

И МАТЕМАТИЧЕСКАЯ

ФИЗИКА

Том 138, № 1

январь, 2004

(C) 2004 г.

И. А. Баталин ${ }^{*}$, И.В. Тютин*

\section{БРСТ-ИНВАРИАНТНАЯ АЛГЕБРА СВЯЗЕЙ В ТЕРМИНАХ КОММУТАТОРОВ И КВАНТОВЫХ АНТИСКОБОК}

На основе формулировки производящих уравнений в дополнительно расширенном фазовом пространстве установлена общая структура БРСТ-инвариантной алгебры связей в коммутаторной и антискобочной формах. Новые переменные гостовского типа обладают относительно квантовой антискобки свойствами полей и антиполей. Приведена явная форма БРСТ-инвариантной калибровочной алгебры для теорий ранга 1 для случаев вейлевского и виковского упорядочений гостовского сектора. Построен унитаризующий гамильтониан в фиксированной калибровке, и показано, что расширенный формализм эквивалентен стандартному БРСТ-БВ

Ключевые слова: БРСТ-симметрия, алгебра связей, квантовые антискобки.

\section{1. ВВЕДЕНИЕ}

При квантовании калибровочных теорий общего вида руководящий принцип состоит в построении фермионного оператора БРСТ-заряда $\Omega$, который удовлетворяет стандартной БРСТ-алгебре $\Omega^{2} \equiv[\Omega, \Omega] / 2=0,\left[G_{C}, \Omega\right]=i \hbar \Omega$, где $G_{C}$ - бозонный оператор гостовского числа [1]-[3]. При разложении в ряд по степеням канонических гостовских переменных $\left(C^{\alpha}, \overline{\mathcal{P}}_{\alpha}\right)$ оператор $\Omega$ имеет вид $\Omega=C^{\alpha} T_{\alpha}+O\left(C^{2} \overline{\mathcal{P}}\right)$, где $T_{\alpha}$ представляют собой исходные связи первого рода, действуюшие как генераторы калибровочной алгебры.

Замечательно, что существуют БРСТ-инвариантные модифицированные связи

$$
\mathcal{T}_{\alpha}=(i \hbar)^{-1}\left[\Omega, \overline{\mathcal{P}}_{\alpha}\right]=T_{\alpha}+O(C \overline{\mathcal{P}})
$$

по построению удовлетворяющие условию $\left[\Omega, \mathcal{T}_{\alpha}\right]=0$, которые, по существу, задают новый предписываемый динамикой набор генераторов калибровочной алгебры. Так как

*Физический институт им. П.Н. Лебедева РАН, Москва, Россия. E-mail: batalin@lpi.ru, tyutin@lpi.ru 
эти генераторы существенно зависят от гостовских операторов $\left(C^{\alpha}, \overline{\mathcal{P}}_{\alpha}\right)$, они определены в расширенном фазовом пространстве, в противоположность исходным связям первого рода $T_{\alpha}$.

Основная идея настояшей работы состоит в переформулировке стандартной БРСТБВФ-схемы квантования непосредственно в терминах БРСТ-инвариантных связей $\mathcal{T}_{\alpha}$ рассматриваемых как новые базисные ингредиенты, посредством дальнейшего расширения фазового пространства, которое прежде описывалось исходными фазовыми переменными и обычными гостами $\left(C^{\alpha}, \overline{\mathcal{P}}_{\alpha}\right)$. Главной мотивацией было ожидание того, что связи $\mathcal{T}_{\alpha}$ будут иметь, вообше говоря, лучшие алгебраические свойства, чем исходные связи $T_{\alpha}$. В частности, как хорошо известно, это происходит в теории бозонной струны (см. [4] и приведенные там ссылки), где алгебра исходных генераторов Вирасоро центрально-расширена, в то время как алгебра соответствуюших БРСТ-инвариантных генераторов совпадает с классической.

Характерным свойством БРСТ-инвариантных генераторов $\mathcal{T}_{\alpha}$ является тот факт, что их алгебра замкнута только в том случае, когда исходные связи первого рода образуют алгебру лиевского типа с постоянными структурными коэффициентами. Оказывается, однако, что алгебра, образуемая генераторами $\mathcal{T}_{\alpha}$ и гостовскими импульсами $\overline{\mathcal{P}}_{\alpha}$, всегда замкнута по построению. Это обстоятельство позволяет сформулировать замкнутую систему производяших уравнений БРСТ-инвариантной калибровочной алгебры в дополнительно расширенном фазовом пространстве. С этой целью мы введем два набора новых канонических пар гостовского типа $\left(B^{\alpha}, \Pi_{\alpha}\right)$ и $\left(B_{\alpha}^{*}, \Pi_{*}^{\alpha}\right)$, которые ведут себя как поля и антиполя относительно квантовых антискобок.

Оказывается, что новые производяшие уравнения БРСТ-инвариантной калибровочной алгебры приводятся к форме операторнозначного мастер-уравнения [5], сформулированного в терминах квантовых антискобок, введенных в работе [6]. Эти квантовые антискобки естественным образом генерируют операторнозначные антиканонические преобразования. Мы опишем их общий вид и трансформационные свойства антискобок, используя дифференциальные уравнения по вспомогательной переменной.

В результате мы получаем два дуальных описания БРСТ-инвариантной калибровочной алгебры в терминах стандартных коммутаторов и квантовых антискобок. Для иллюстрации этого дуализма в техническом отношении подробно рассмотрен случай теорий ранга 1 с вейлевским и виковским упорядочением гостовского сектора.

Как обычно, $\varepsilon(f) \equiv \varepsilon_{f}$ означает грассманову четность величины $f,[f, g]$ означает стандартный суперкоммутатор $[f, g] \equiv f g-(-1)^{\varepsilon_{f}} \varepsilon_{g} g f$ любых двух операторов $f$ и $g$. Он удовлетворяет стандартному правилу Лейбница, $[f g, h]=f[g, h]+[f, h] g(-1)^{\varepsilon_{g} \varepsilon_{h}}$, и тождеству Якоби, $[f,[g, h]](-1)^{\varepsilon_{f} \varepsilon_{h}}+\operatorname{cycle}(f, g, h)=0$. Смысл других обозначений ясен из контекста.

\section{2. КВАНТОВЫЕ АНТИСКОБКИ И АНТИКАНОНИЧЕСКИЕ ПРЕОБРАЗОВАНИЯ}

Здесь мы напомним определения и свойства квантовых антискобок, введенных в работах $[6],[7]$. Затем определим операторнозначные антиканонические преобразования 
и выведем трансформационные свойства антискобок относительно антиканонических преобразований их аргументов.

Пусть $Q$ - фермионный нильпотентньй оператор,

$$
\varepsilon(Q)=1, \quad Q^{2} \equiv \frac{1}{2}[Q, Q]=0 .
$$

Тогда квантовая антискобка любых двух операторов $f$ и $g$ определяется формулой

$$
(f, g)_{Q} \equiv \frac{1}{2}\left([f,[Q, g]]-[g,[Q, f]](-1)^{\left(\varepsilon_{f}+1\right)\left(\varepsilon_{g}+1\right)}\right) .
$$

Она обладает следующими свойствами:

$$
\begin{gathered}
\varepsilon\left((f, g)_{Q}\right)=\varepsilon_{f}+\varepsilon_{g}+1, \\
(f, g)_{Q}=-(g, f)_{Q}(-1)^{\left(\varepsilon_{f}+1\right)\left(\varepsilon_{g}+1\right)} .
\end{gathered}
$$

Из определения (2.2) следует, что справедливы модифицированные правило Лейбница

$$
\begin{aligned}
& (f g, h)_{Q}-f(g, h)_{Q}-(f, h)_{Q} g(-1)^{\varepsilon_{g}\left(\varepsilon_{h}+1\right)}= \\
& \quad=\frac{1}{2}\left([f, h][g, Q](-1)^{\varepsilon_{h}\left(\varepsilon_{g}+1\right)}+[f, Q][g, h](-1)^{\varepsilon_{g}}\right)
\end{aligned}
$$

и тождество Якоби

$$
\left(f,(g, h)_{Q}\right)_{Q}(-1)^{\left(\varepsilon_{f}+1\right)\left(\varepsilon_{h}+1\right)}+\operatorname{cycle}(f, g, h)=-\frac{1}{2}\left[(f, g, h)_{Q}(-1)^{\left(\varepsilon_{f}+1\right)\left(\varepsilon_{h}+1\right)}, Q\right],
$$

где равенство

$$
\begin{aligned}
(f, g, h)_{Q} & \equiv \frac{1}{3}(-1)^{\left(\varepsilon_{f}+1\right)\left(\varepsilon_{h}+1\right)}\left(\left[(f, g)_{Q}, h\right](-1)^{\varepsilon_{h}+\left(\varepsilon_{f}+1\right)\left(\varepsilon_{h}+1\right)}+\operatorname{cycle}(f, g, h)\right)= \\
& =\frac{1}{3}(-1)^{\left(\varepsilon_{f}+1\right)\left(\varepsilon_{h}+1\right)}\left(\left[f,(g, h)_{Q}\right](-1)^{\varepsilon_{g}+\left(\varepsilon_{f}+1\right)\left(\varepsilon_{h}+1\right)}+\operatorname{cycle}(f, g, h)\right)
\end{aligned}
$$

определяет следуюшую, 3 -антискобку для любых операторов $f, g, h$.

В свою очередь, 3-антискобка (2.7) удовлетворяет следуюшему тождеству, включающему следуюшую, 4-антискобку, и т.д. В работах [7] эта иерархия последовательных квантовых антискобок высших порядков полностью определена с помошью соответствующего производящего механизма.

Пусть $B$ - бозонный оператор и $A$ - произвольный оператор. Из (2.6) получаем

$$
\begin{aligned}
\left(B,(B, A)_{Q}\right)_{Q} & =\frac{1}{2}\left((B, B)_{Q}, A\right)_{Q}-\frac{1}{4}\left[(B, B, A)_{Q}, Q\right], \\
(B, B, A)_{Q} & =\frac{1}{3}\left(-\left[A,(B, B)_{Q}\right]+2\left[(A, B)_{Q}, B\right]\right), \\
\left(B,(B, B)_{Q}\right)_{Q} & =\frac{1}{6}\left[\left[B,(B, A)_{Q}\right], Q\right] .
\end{aligned}
$$


Другое важное следствие определения (2.2) и условия нильпотентности (2.1) есть

$$
\left[Q,(f, g)_{Q}\right]=[[Q, f],[Q, g]]
$$

Определим операторнозначное антиканоническое преобразование следующим образом. Пусть $A_{0}$ - произвольный исходный оператор, а $X, \varepsilon(X)=1,-$ фермионный антиканонический генератор. Тогда уравнение

$$
\frac{d A(\lambda)}{d \lambda}=(X, A(\lambda))_{Q}, \quad A(0)=A_{0},
$$

определяет антиканоническое преобразование $A_{0} \rightarrow A$.

Из (2.11), (2.12) следует

$$
\frac{d}{d \lambda}[Q, A]=[[Q, X],[Q, A]]
$$

откуда видно, что $[Q, X]$ представляет собой бозонный генератор канонического преобразования $\left[Q, A_{0}\right]$. Общее решение параметрического дифференшиального уравнения (2.12) есть

$$
A=\tilde{A}+[Q, Y]
$$

где

$$
\begin{gathered}
\tilde{A} \equiv \tilde{A}(\lambda)=e^{\lambda[Q, X]} A_{0} e^{-\lambda[Q, X]}, \\
Y=-\frac{1}{2} \int_{0}^{\lambda} d \lambda^{\prime} e^{\left(\lambda-\lambda^{\prime}\right)[Q, X] / 2}\left[X, \tilde{A}\left(\lambda^{\prime}\right)\right] e^{-\left(\lambda-\lambda^{\prime}\right)[Q, X] / 2} .
\end{gathered}
$$

Сушествует красивая интерпретация решения (2.14): первый член в правой части, $\tilde{A}$, представляет собой каноническое преобразование $A_{0}$ с генератором $[Q, X]$, в то время как второй член, $[Q, Y]$, есть “точная" форма. Вычисляя выражение $(2.14)$ в первом порядке по $X$,

$$
A-A_{0}=\lambda\left(\left[[Q, X], A_{0}\right]-\frac{1}{2}[Q,[X, A]]\right)+O\left(\lambda^{2}\right)=\lambda\left(X, A_{0}\right)_{Q}+O\left(\lambda^{2}\right),
$$

мы видим, что часть $\left[[Q, X], A_{0}\right]$ квантовой антискобки $\left(X, A_{0}\right)_{Q}$ есть в точности инфиинитезимальное каноническое преобразование $A_{0}$ с оператором $[Q, X]$ в качестве генератора, в то время как слагаемое $(-[Q,[X, A]] / 2)$ (“точная" часть) представляет собой отклонение от унимодулярности, характерное для антиканонических преобразований.

Пусть $A$ и $B$ - антиканонические преобразования $A_{0}$ и $B_{0}$ согласно уравнению (2.12). Тогда квантовая антискобка $(A, B)_{Q}$ удовлетворяет уравнению

$$
\frac{d}{d \lambda}(A, B)_{Q}=\left(X,(A, B)_{Q}\right)_{Q}+\frac{1}{2}\left[(X, A, B)_{Q}, Q\right],
$$


где учтено модифицированное тождество Якоби (2.6) для $f=X, g=A, h=B$. Из (2.18) следует, что отклонение трансформационных свойств антискобки $(A, B)_{Q}$ от канонических (решения однородной части уравнения (2.18)) дается “точной” формой

$$
\left[\frac{1}{2} \int_{0}^{\lambda} d \lambda^{\prime} e^{\left(\lambda-\lambda^{\prime}\right)[Q, X] / 2}(X, A, B)_{Q} e^{-\left(\lambda-\lambda^{\prime}\right)[Q, X] / 2}, Q\right]
$$

Таким образом, мы видим, что появление ненулевой “точной” формы $\left[(f, g, h)_{Q}, Q\right]$, которая деформирует сильное тождество Якоби до модифицированной формы, приводит к аналогичной деформации инвариантных свойств квантовой антискобки относительно антиканонического преобразования ее аргументов.

\section{3. БРСТ-ИНВАРИАНТНАЯ АЛГЕБРА СВЯЗЕЙ}

Пусть $\Omega$ - фермионный оператор, удовлетворяющий стандартной БРСТ-алгебре

$$
[\Omega, \Omega]=0, \quad\left[G_{C}, \Omega\right]=i \hbar \Omega
$$

где $G_{C}$ - бозонный оператор гостовского числа.

Для определенности мы предположим, что гостовский сектор содержит канонические пары $\left(C^{\alpha}, \overline{\mathcal{P}}_{\alpha}\right), \varepsilon\left(C^{\alpha}\right)=\varepsilon\left(\overline{\mathcal{P}}_{\alpha}\right)=\varepsilon_{\alpha}+1$, с ненулевыми коммутаторами

$$
\left[C^{\alpha}, \overline{\mathcal{P}}_{\beta}\right]=i \hbar \delta_{\beta}^{\alpha}
$$

и БРСТ-оператор $\Omega C \overline{\mathcal{P}}$-упорядочен. Кроме того, мы примем следуюшее предписание для значений гостовских чисел:

$$
\left[G_{C}, C^{\alpha}\right]=i \hbar C^{\alpha}, \quad\left[G_{C}, \overline{\mathcal{P}}_{\alpha}\right]=-i \hbar \overline{\mathcal{P}}_{\alpha}
$$

что соответствует неприводимым теориям. БРСТ-инвариантные связи определяются следующим образом:

$$
\mathcal{T}_{\alpha}=(i \hbar)^{-1}\left[\Omega, \overline{\mathcal{P}}_{\alpha}\right], \quad\left[\Omega, \mathcal{T}_{\alpha}\right]=0
$$

В терминах квантовой антискобки (2.2) с заменой $Q$ на $\Omega$ мы имеем следуюшие соотношения:

$$
\begin{aligned}
\left(\mathcal{T}_{\alpha}, \mathcal{T}_{\beta}\right)_{\Omega} & =0 \\
\left(\overline{\mathcal{P}}_{\alpha}, \overline{\mathcal{P}}_{\beta}\right)_{\Omega} & =(i \hbar)^{2} U_{\alpha \beta}^{\gamma} \overline{\mathcal{P}}_{\gamma}(-1)^{\varepsilon_{\alpha}+\varepsilon_{\beta}+\varepsilon_{\gamma}} \\
\left(\overline{\mathcal{P}}_{\alpha}, \mathcal{T}_{\beta}\right)_{\Omega} & =\frac{1}{2} i \hbar\left[\mathcal{T}_{\alpha}, \mathcal{T}_{\beta}\right](-1)^{\varepsilon_{\alpha}}
\end{aligned}
$$

где операторнозначные структурные коэффициенты

$$
U_{\alpha \beta}^{\gamma}=-U_{\beta \alpha}^{\gamma}(-1)^{\varepsilon_{\alpha} \varepsilon_{\beta}},
$$

вообще говоря, зависят от гостов. 
Если мы представим оператор $\Omega$ явно в виде $C \overline{\mathcal{P}}$-упорядоченного разложения по степеням гостов с учетом $(3.1),(3.3)$,

$$
\Omega=C^{\alpha} T_{\alpha}+\sum_{n \geqslant 1} \frac{1}{n !(n+1) !} C^{\alpha_{n+1}} \ldots C^{\alpha_{1}} \Omega_{\alpha_{1} \ldots \alpha_{n+1}}^{\beta_{n} \ldots \beta_{1}} \overline{\mathcal{P}}_{\beta_{1}} \ldots \overline{\mathcal{P}}_{\beta_{n}}
$$

то соответствуюшие разложения операторов $\mathcal{T}_{\alpha}$ и $U_{\alpha \beta}^{\gamma}$ суть

$$
\begin{gathered}
\mathcal{T}_{\alpha}=T_{\alpha}+\sum_{n \geqslant 1} \frac{1}{(n !)^{2}} C^{\alpha_{n}} \ldots C^{\alpha_{1}} \Omega_{\alpha_{1} \ldots \alpha_{n} \alpha}^{\beta_{n} \ldots \beta_{1}} \overline{\mathcal{P}}_{\beta_{1}} \ldots \overline{\mathcal{P}}_{\beta_{n}}, \\
U_{\alpha \beta}^{\gamma}=\sum_{n \geqslant 0} \frac{1}{n !(n+1) !} C^{\alpha_{n}} \ldots C^{\alpha_{1}} \Omega_{\alpha_{1} \ldots \alpha_{n} \alpha \beta}^{\gamma \beta_{n} \ldots \beta_{1}} \overline{\mathcal{P}}_{\beta_{1}} \ldots \overline{\mathcal{P}}_{\beta_{n}}(-1)^{\varepsilon_{\beta}+\varepsilon_{\gamma}} .
\end{gathered}
$$

Используя свойство (2.11) и определение (3.4), мы получаем следуюшую коммутаторную алгебру:

$$
\begin{aligned}
{\left[\mathcal{T}_{\alpha}, \mathcal{T}_{\beta}\right] } & =i \hbar U_{\alpha \beta}^{\gamma} \mathcal{T}_{\gamma}-\left[U_{\alpha \beta}^{\gamma}, \Omega\right] \overline{\mathcal{P}}_{\gamma}, \\
{\left[\overline{\mathcal{P}}_{\alpha}, \overline{\mathcal{P}}_{\beta}\right] } & =0 \\
{\left[\overline{\mathcal{P}}_{\alpha}, \mathcal{T}_{\beta}\right] } & =(i \hbar)^{-1}\left(\overline{\mathcal{P}}_{\alpha}, \overline{\mathcal{P}}_{\beta}\right)_{\Omega} .
\end{aligned}
$$

Таким образом, мы видим, что БРСТ-инвариантные связи $\mathcal{T}_{\alpha}$ вместе с гостовскими импульсами $\overline{\mathcal{P}}_{\alpha}$ образуют две дуальные операторные алгебры, а именно квантово-антискобочную алгебру (3.5) и коммутаторную алгебру (3.9).

\section{4. ПРОИЗВОДЯШИЕ УРАВНЕНИЯ БРСТ-ИНВАРИАНТНОЙ АЛГЕБРЫ СВЯЗЕЙ}

Так как мы установили, что $\mathcal{T}_{\alpha}$ вместе с $\overline{\mathcal{P}}_{\alpha}$ образуют две дуальные алгебры, кажется совершенно естественным сформулировать соответствуюшие производящие уравнения в духе обшей идеологии БРСТ-БВФ-подхода. Мы можем рассматривать $\mathcal{T}_{\alpha}$ и $\overline{\mathcal{P}}_{\alpha}$ как связи первого рода, а формулы (3.9) как их соотношения инволюции. Мы можем поворачивать эти связи первого рода несингулярными операторными матрицами, так что кажется естественным несколько обобщить определение $\mathcal{T}_{\alpha}$.

Прежде всего, повернем $\overline{\mathcal{P}}_{\alpha}$ в соотношении (3.4),

$$
\overline{\mathcal{P}}_{\alpha} \rightarrow X_{\alpha}=\Lambda_{\alpha}^{\beta} \overline{\mathcal{P}}_{\beta}
$$

так что новые связи $\mathcal{T}_{\alpha}$ суть

$$
\mathcal{T}_{\alpha}=(i \hbar)^{-1}\left[\Omega, X_{\alpha}\right], \quad\left[G_{C}, X_{\alpha}\right]=-i \hbar X_{\alpha} .
$$

Эти $\mathcal{T}_{\alpha}$, однако, остаются сильно БРСТ-инвариантными, $\left[\Omega, \mathcal{T}_{\alpha}\right]=0$. Чтобы ослабить инвариантность, мы можем еше более модифицировать определение $\mathcal{T}_{\alpha}$ :

$$
\mathcal{T}_{\alpha}=(i \hbar)^{-1}\left[\Omega, X_{\alpha}\right]-V_{\alpha}^{\beta} X_{\beta}(-1)^{\varepsilon_{\alpha}+\varepsilon_{\beta}},
$$


где $V_{\alpha}^{\beta}$ есть плоская БРСТ-связность,

$$
R_{\alpha}^{\beta} \equiv(i \hbar)^{-1}\left[\Omega, V_{\alpha}^{\beta}\right]-V_{\alpha}^{\gamma} V_{\gamma}^{\beta}(-1)^{\varepsilon_{\alpha}+\varepsilon_{\gamma}}=0 .
$$

В этом случае мы имеем слабую БРСТ-инвариантность,

$$
\left[\Omega, \mathcal{T}_{\alpha}\right]=i \hbar V_{\alpha}^{\beta} \mathcal{T}_{\beta},
$$

которая соответствует повороту

$$
\mathcal{T}_{\alpha} \rightarrow G_{\alpha}^{\beta} \mathcal{T}_{\beta}, \quad X_{\alpha} \rightarrow G_{\alpha}^{\beta} X_{\beta}(-1)^{\varepsilon_{\alpha}+\varepsilon_{\beta}}
$$

в (4.2) и выбору

$$
V_{\alpha}^{\beta}=(i \hbar)^{-1}\left[\Omega, G_{\alpha}^{\gamma}\right] G_{\gamma}^{-1 \beta} .
$$

Мы ожидаем, что такие повороты (4.1)-(4.6) будут частью естественного произвола в обшем решении производяших уравнений алгебры.

Перейдем непосредственно к формулировке нужных нам производящих уравнений. Начнем с некоторых операторов $\mathcal{T}_{\alpha}$ и $X_{\alpha}$, определенных в том же расширенном фазовом пространстве, что и БРСТ-оператор $\Omega$. Их грассмановы четности и значения внутренних гостовских чисел имеют вид

$$
\begin{aligned}
\varepsilon\left(\mathcal{T}_{\alpha}\right) & =\varepsilon_{\alpha}, & \varepsilon\left(X_{\alpha}\right) & =\varepsilon_{\alpha}+1, \\
{\left[G_{C}, \mathcal{T}_{\alpha}\right] } & =0, & {\left[G_{C}, X_{\alpha}\right] } & =-i \hbar X_{\alpha} .
\end{aligned}
$$

Затем мы еше расширяем фазовое пространство, вводя новые канонические пары гостовского типа посредством соответствия

$$
\mathcal{T}_{\alpha} \mapsto\left(B^{\alpha}, \Pi_{\alpha}\right), \quad X_{\alpha} \mapsto\left(B_{\alpha}^{*}, \Pi_{*}^{\alpha}\right)
$$

с ненулевыми коммутаторами

$$
\left[B^{\alpha}, \Pi_{\beta}\right]=i \hbar \delta_{\beta}^{\alpha}, \quad\left[B_{\alpha}^{*}, \Pi_{*}^{\beta}\right]=i \hbar \delta_{\alpha}^{\beta} .
$$

Их грассмановы четности суть

$$
\varepsilon\left(B^{\alpha}\right)=\varepsilon\left(\Pi_{\alpha}\right)=\varepsilon_{\alpha}+1, \quad \varepsilon\left(B_{\alpha}^{*}\right)=\varepsilon\left(\Pi_{*}^{\alpha}\right)=\varepsilon_{\alpha} .
$$

Все новые операторы коммутируют с оператором внутреннего гостовского числа $G_{C}$. Однако они имеют свои собственные операторы гостовского числа $G_{B}$ и $G_{B^{*}}$,

$$
\begin{gathered}
{\left[G_{B}, B^{\alpha}\right]=i \hbar B^{\alpha}, \quad\left[G_{B}, \Pi_{\alpha}\right]=-i \hbar \Pi_{\alpha},} \\
{\left[G_{B^{*}}, B_{\alpha}^{*}\right]=i \hbar B_{\alpha}^{*}, \quad\left[G_{B^{*}}, \Pi_{*}^{\alpha}\right]=-i \hbar \Pi_{*}^{\alpha},} \\
{\left[G_{B^{*}}, B^{\alpha}\right]=\left[G_{B^{*}}, \Pi_{\alpha}\right]=\left[G_{B}, B_{\alpha}^{*}\right]=\left[G_{B}, \Pi_{*}^{\alpha}\right]=0,} \\
{\left[G_{B}, G_{B^{*}}\right]=\left[G_{C}, G_{B}\right]=\left[G_{C}, G_{B^{*}}\right]=0 .}
\end{gathered}
$$


Полный оператор гостовского числа равен

$$
G=G_{C}+G_{B}-2 G_{B^{*}}=G_{C B^{*}}+G_{B B^{*}}
$$

где

$$
G_{C B^{*}}=G_{C}-G_{B^{*}}, \quad G_{B B^{*}}=G_{B}-G_{B^{*}} .
$$

Пусть $A$ - произвольный оператор. Определим значения полного гостовского числа, $\operatorname{gh}(A)$, и полной степени, $\operatorname{deg}(A)$, следуюшим образом:

$$
[G, A]=i \hbar \operatorname{gh}(A) A, \quad\left[G_{B B^{*}}, A\right]=i \hbar \operatorname{deg}(A) A,
$$

так что

$$
\left[G_{C B^{*}}, A\right]=i \hbar(\operatorname{gh}(A)-\operatorname{deg}(A)) A .
$$

В частности, мы имеем

$$
\varepsilon(\Omega)=1, \quad \operatorname{gh}(\Omega)=1, \quad \operatorname{deg}(\Omega)=0 .
$$

Для дальнейшего рассмотрения удобно ввести конденсированные обозначения

$$
\begin{gathered}
T_{A} \equiv\left\{\mathcal{T}_{\alpha} ;-X_{\alpha}\right\} \\
C^{A} \equiv\left\{B^{\alpha} ; \Pi_{*}^{\alpha}(-1)^{\varepsilon_{\alpha}+1}\right\}, \quad \overline{\mathcal{P}}_{A} \equiv\left\{\Pi_{\alpha} ; B_{\alpha}^{*}\right\}, \quad\left[C^{A}, \overline{\mathcal{P}}_{B}\right]=i \hbar \delta_{B}^{A} .
\end{gathered}
$$

Имеем

$$
\begin{gathered}
\varepsilon\left(T_{A}\right)=\left\{\varepsilon_{\alpha} ; \varepsilon_{\alpha}+1\right\}, \quad \operatorname{gh}\left(T_{A}\right)=\{0 ;-1\}, \quad \operatorname{deg}\left(T_{A}\right)=\{0 ; 0\}, \\
\varepsilon\left(C^{A}\right)=\left\{\varepsilon_{\alpha}+1 ; \varepsilon_{\alpha}\right\}, \quad \operatorname{gh}\left(C^{A}\right)=\{1 ; 2\}, \quad \operatorname{deg}\left(C^{A}\right)=\{1 ; 1\}, \\
\varepsilon\left(T_{A}\right) \equiv \varepsilon_{A}, \quad \varepsilon\left(\overline{\mathcal{P}}_{A}\right)=\varepsilon\left(C^{A}\right)=\varepsilon_{A}+1, \\
\operatorname{gh}\left(\overline{\mathcal{P}}_{A}\right)=-\operatorname{gh}\left(C^{A}\right), \quad \operatorname{deg}\left(\overline{\mathcal{P}}_{A}\right)=-\operatorname{deg}\left(C^{A}\right) .
\end{gathered}
$$

В новом расширенном фазовом пространстве, координатами которого являются исходные фазовые переменные, обычные госты и новые переменные (4.10), мы рассмотрим следуюшую систему уравнений:

$$
\begin{aligned}
& {\left[\Sigma_{1}, \Sigma_{1}\right]=0, \quad[\Delta, \Delta]=0, \quad\left[\Delta, \Sigma_{1}\right]=0,} \\
& \varepsilon\left(\Sigma_{1}\right)=1, \quad \operatorname{gh}\left(\Sigma_{1}\right)=1, \quad \operatorname{deg}\left(\Sigma_{1}\right)=1, \\
& \varepsilon(\Delta)=1, \quad \operatorname{gh}(\Delta)=1, \quad \operatorname{deg}(\Delta)=0,
\end{aligned}
$$

вместе с граничными условиями

$$
\Sigma_{1}=C^{A} T_{A}+\cdots, \quad \Delta=\Omega+\cdots,
$$


где многоточием обозначены все возможные члены высшего порядка по $\left(C^{A}, \overline{\mathcal{P}}_{A}\right)$, совместные с (4.29), (4.30). Мы также потребуем, чтобы оператор $\Delta$ удовлетворял дополнительному условию: матрица $\Delta$-антискобок $\left(B^{\alpha}, B_{\beta}^{*}\right)_{\Delta}$ должна быть обратима.

Мы утверждаем, что уравнения (4.28)-(4.31), разложенные в ряд по $\left(C^{A}, \overline{\mathcal{P}}_{A}\right)$, генерируют БРСТ-инвариантную алгебру связей. Чтобы увидеть это, рассмотрим $C \overline{\mathcal{P}}$-упорядоченное разложение $\Sigma_{1}$ и $\Delta$,

$$
\begin{gathered}
\Sigma_{1}=C^{A} T_{A}+\frac{1}{2} C^{B} C^{A} U_{A B}^{C} \overline{\mathcal{P}}_{C}(-1)^{\varepsilon_{B}+\varepsilon_{C}}+\cdots \\
\Delta=\Omega+C^{A} V_{A}^{B} \overline{\mathcal{P}}_{B}(-1)^{\varepsilon_{B}}+\frac{1}{4} C^{B} C^{A} V_{A B}^{C D} \overline{\mathcal{P}}_{D} \overline{\mathcal{P}}_{C}(-1)^{\varepsilon_{B}+\varepsilon_{D}}+\cdots
\end{gathered}
$$

Подставляя (4.32) в первое уравнение $(4.28)$, мы получаем во втором порядке по $C^{A}$ стандартные соотношения инволюции

$$
\left[T_{A}, T_{B}\right]=i \hbar U_{A B}^{C} T_{C}
$$

Далее после подстановки (4.33) во второе уравнение (4.28) имеем в нулевом и первом порядках по $C^{A}$

$$
[\Omega, \Omega]=0
$$

и

$$
\left[V_{A}^{B}, \Omega\right](-1)^{\varepsilon_{B}}=i \hbar V_{A}^{C} V_{C}^{B} .
$$

Аналогично, подставляя (4.32), (4.33) в третье уравнение (4.28), мы получаем в первом порядке по $C^{A}$

$$
\left[T_{A}, \Omega\right]=-i \hbar V_{A}^{B} T_{B}
$$

В уравнении (4.35) мы узнаем условие нильпотентности для $\Omega$. Замечательно, что уравнение (4.36) есть не что иное, как условие нильпотентности матрично-расширенного $\Omega$, $\widehat{\Omega}_{A}^{B}$,

$$
\widehat{\Omega}_{A}^{C} \widehat{\Omega}_{C}^{B}=0, \quad \widehat{\Omega}_{A}^{B} \equiv(-1)^{\varepsilon} A \delta_{A}^{B} \Omega-i \hbar V_{A}^{B} .
$$

Соотношения инволюции (4.37) по своей сути означают БРСТ-инвариантность связей $T_{A}$ в наиболее общей (слабой) форме.

Нетрудно видеть, что приведенные выше (частные) представления (4.2), (4.3), (4.5) немедленно вытекают из (4.37), если $V_{A}^{B}$ выбрать в виде

$$
V_{A}^{B}=\left(\begin{array}{cc}
V_{\alpha}^{\beta}(-1)^{\varepsilon_{\alpha}} & 0 \\
\delta_{\alpha}^{\beta}(-1)^{\varepsilon_{\alpha}} & -V_{\alpha}^{\beta}(-1)^{\varepsilon_{\beta}}
\end{array}\right) .
$$

Рассмотрим теперь второе уравнение (4.28) во втором порядке по $C^{A}$. Мы имеем

$$
\begin{aligned}
& {\left[V_{A}^{C}, V_{B}^{D}\right](-1)^{\left(\varepsilon_{B}+1\right)\left(\varepsilon_{C}+1\right)}-(A \leftrightarrow B)(-1)^{\varepsilon_{A} \varepsilon_{B}}=} \\
& =i \hbar\left(V_{A}^{E} V_{E B}^{C D}(-1)^{\varepsilon_{B}}-(A \leftrightarrow B)(-1)^{\varepsilon_{A} \varepsilon_{B}}\right)+ \\
& +i \hbar\left(V_{A B}^{C E} V_{E}^{D}(-1)^{\varepsilon_{C}}-(C \leftrightarrow D)(-1)^{\varepsilon_{C} \varepsilon_{D}}\right)- \\
& -\left[V_{A B}^{C D}, \Omega\right](-1)^{\varepsilon_{C}+\varepsilon_{D}}-\frac{1}{2}(i \hbar)^{2} V_{A B}^{E F} V_{F E}^{C D} \text {. }
\end{aligned}
$$


В том же самом порядке по $C^{A}$ третье уравнение (4.28) дает

$$
\begin{aligned}
& \left(\left[T_{A}, V_{B}^{C}\right]-(A \leftrightarrow B)(-1)^{\varepsilon_{A} \varepsilon_{B}}\right)-i \hbar U_{A B}^{D} V_{D}^{C}+ \\
& \quad+i \hbar\left(V_{A}^{D} U_{D B}^{C}(-1)^{\varepsilon_{B}}-(A \leftrightarrow B)(-1)^{\varepsilon_{A} \varepsilon_{B}}\right)+ \\
& \quad+\left[U_{A B}^{C}, \Omega\right](-1)^{\varepsilon_{C}}+\frac{1}{2} i \hbar V_{A B}^{E D} Z_{D E}^{C}=0
\end{aligned}
$$

где

$$
\begin{gathered}
Z_{A B}^{C} \equiv T_{A} \delta_{B}^{C}-T_{B} \delta_{A}^{C}(-1)^{\varepsilon_{A} \varepsilon_{B}}-i \hbar U_{A B}^{C}, \\
Z_{A B}^{C} T_{C}=0 .
\end{gathered}
$$

Умножая (4.41) на $T_{C}$ справа, мы получаем тождественный ноль благодаря соотношениям (4.34), (4.37).

В разложении (4.28) с точностью до третьего порядка по $C^{A}$ других уравнений не возникает.

Если заданы связи первого рода $T_{A}$, из уравнения (4.34) определяются $U_{A B}^{C}$. Далее уравнения (4.36), (4.37) определяют $V_{B}^{A}$. Затем уравнения $(4.40),(4.41)$ определяют $V_{A B}^{C D}$ и т.д.

Если производяшие уравнения (4.28) допускают решение для $\Delta$, линейное по $C^{A}$,

$$
\Delta=\Omega+C^{A} V_{A}^{B} \overline{\mathcal{P}}_{B}(-1)^{\varepsilon_{B}},
$$

что в соответствии с (4.40), (4.41) подразумевает

$$
\left[V_{A}^{C}, V_{B}^{D}\right](-1)^{\left(\varepsilon_{B}+1\right)\left(\varepsilon_{C}+1\right)}-(A \leftrightarrow B)(-1)^{\varepsilon_{A} \varepsilon_{B}}=0
$$

$$
\begin{aligned}
\left(\left[T_{A}, V_{B}^{C}\right]-\right. & \left.(A \leftrightarrow B)(-1)^{\varepsilon_{A} \varepsilon_{B}}\right)-i \hbar U_{A B}^{D} V_{D}^{C}+ \\
& +i \hbar\left(V_{A}^{D} U_{D B}^{C}(-1)^{\varepsilon_{B}}-(A \leftrightarrow B)(-1)^{\varepsilon_{A} \varepsilon_{B}}\right)+\left[U_{A B}^{C}, \Omega\right](-1)^{\varepsilon_{C}}=0,
\end{aligned}
$$

то для любых величин $f, g, h$, зависяших только от $B^{\alpha}, B_{\alpha}^{*}$, их $\Delta$-антискобки обладают свойством

$$
\left[(f, g)_{\Delta}, h\right]=0
$$

так что их 3 -антискобки исчезают,

$$
(f, g, h)_{\Delta}=0,
$$

и поэтому тождество Якоби становится сильным. Поскольку $f, g, h$ коммутируют между собой, правило Лейбница (2.5) также становится сильным. Кроме того, согласно предписанию (4.12)-(4.15) мы имеем

$$
\begin{aligned}
\varepsilon\left(B^{\alpha}\right)+\varepsilon\left(B_{\alpha}^{*}\right) & =1, \\
\operatorname{gh}\left(B^{\alpha}\right)+\operatorname{gh}\left(B_{\alpha}^{*}\right) & =-1, \\
\operatorname{deg}\left(B^{\alpha}\right)+\operatorname{deg}\left(B_{\alpha}^{*}\right) & =0 .
\end{aligned}
$$


Таким образом, из уравнений (4.48)-(4.51) следует, что переменные $B^{\alpha}$ и $B_{\alpha}^{*}$ ведут себя как нормальные поля и антиполя относительно $\Delta$-антискобки при условии, что соотношения (4.45), (4.46) удовлетворены.

Подчеркнем, однако, что не требуется, чтобы условия (4.45), (4.46) обязательно выполнялись. Они только выделяют определенный базис связей $T_{A}$ и величин $V_{A}^{B}$, в котором переменные $B^{\alpha}, B_{\alpha}^{*}$ допускают простую интерпретацию. В обшем случае коэффициенты $V_{A B}^{C D}$ отличны от нуля и разложения (4.32), (4.33) включают все высшие порядки по гостам. Поэтому, вообще говоря, $\Delta$-антискобки не удовлетворяют сильному тождеству Якоби, даже если их аргументы зависят только от $B^{\alpha}, B_{\alpha}^{*}$.

В принципе соотношения инволюции (4.34)-(4.37) являются единственными условиями, которым должны удовлетворять члены низших порядков в (4.32), (4.33). Однако мы требуем дополнительно, чтобы правая часть (4.37) была разрешима относительно $\mathcal{T}_{\alpha}$ : это есть в точности дополнительное условие, сформулированное ниже формул (4.31). Это условие означает, что любые связи $T_{A}$, удовлетворяюшие соотношениям инволюции, могут быть повернуты несингулярной матрищей к форме $T_{A}=\left\{(i \hbar)^{-1}\left[\Omega, \overline{\mathcal{P}}_{\alpha}\right] ;-\overline{\mathcal{P}}_{\alpha}\right\}$.

\section{5. ПРОИЗВОДЯШИЕ УРАВНЕНИЯ АНТИСКОБОЧНОЙ АЛГЕБРЫ}

Как мы видели выше, переменные $B^{\alpha}$ и $B_{\alpha}^{*}$ ведут себя как поля и антиполя относительно $\Delta$-антискобки. Кажется совершенно естественным ожидать подобного поведения импульсов $\Pi_{*}^{\alpha}$ и $\Pi_{\alpha}$ относительно некоторой “дуальной” антискобки.

Для реализации этой идеи определим резольвентный оператор $\bar{\Delta}$ как решение производящих уравнений

$$
\begin{gathered}
{[\bar{\Delta}, \bar{\Delta}]=0, \quad[\Delta, \bar{\Delta}]=i \hbar G_{B B^{*}},} \\
\varepsilon(\bar{\Delta})=1, \quad \operatorname{gh}(\bar{\Delta})=-1, \quad \operatorname{deg}(\bar{\Delta})=0,
\end{gathered}
$$

с граничными условиями

$$
\bar{\Delta}=\bar{\Omega}+\cdots,
$$

где многоточием обозначены все члены высших порядков по переменным $\left(C^{A}, \overline{\mathcal{P}}_{A}\right)$, допускаемые свойствами (5.2), а $\bar{\Omega}$ является членом нулевого порядка.

Рассмотрим $C \overline{\mathcal{P}}$-упорядоченное разложение $\bar{\Delta}$ в степенной ряд

$$
\bar{\Delta}=\bar{\Omega}+C^{A} \bar{V}_{A}^{B} \overline{\mathcal{P}}_{B}(-1)^{\varepsilon_{B}}+\frac{1}{4} C^{B} C^{A} \bar{V}_{A B}^{C D} \overline{\mathcal{P}}_{D} \overline{\mathcal{P}}_{C}(-1)^{\varepsilon_{B}+\varepsilon_{D}}+\cdots,
$$

аналогичное (4.33). Из уравнений (5.1), (5.2) мы получаем следуюшие уравнения низших порядков для операторных коэффициентов:

$$
\begin{aligned}
& {[\bar{\Omega}, \bar{\Omega}] }=0, \\
& {\left[\bar{V}_{A}^{B}, \bar{\Omega}\right](-1)^{\varepsilon_{B}} }=i \hbar \bar{V}_{A}^{C} \bar{V}_{C}^{B}, \\
& {[\Omega, \bar{\Omega}] }=0, \\
& {\left[V_{A}^{B}, \bar{\Omega}\right](-1)^{\varepsilon_{B}}+\left[\bar{V}_{A}^{B}, \Omega\right](-1)^{\varepsilon_{B}}+i \hbar \delta_{A}^{B}=i \hbar V_{A}^{C} \bar{V}_{C}^{B}+i \hbar \bar{V}_{A}^{C} V_{C}^{B} . }
\end{aligned}
$$


В терминах операторнозначной матрицы (4.38) и аналогичной матрицы для $\bar{\Omega}$,

$$
\widehat{\bar{\Omega}}_{A}^{B} \equiv(-1)^{\varepsilon_{A}} \delta_{A}^{B} \bar{\Omega}-i \hbar \bar{V}_{A}^{B},
$$

уравнения (5.5)-(5.8) переписываются в виде

$$
\begin{aligned}
\hat{\bar{\Omega}}_{A}^{C} \widehat{\bar{\Omega}}_{C}^{B} & =0, \\
\widehat{\Omega}_{A}^{C} \widehat{\bar{\Omega}}_{C}^{B}+\widehat{\bar{\Omega}}_{A}^{C} \widehat{\Omega}_{C}^{B} & =i \hbar \delta_{A}^{B} .
\end{aligned}
$$

Нильпотентный оператор $\bar{\Omega}$ определен в том же самом пространстве, что и $\Omega$, и его разложение в ряд по обычным гостам $\left(C^{\alpha}, \overline{\mathcal{P}}_{\alpha}\right)$ начинается с члена $\bar{T}^{\alpha} \overline{\mathcal{P}}_{\alpha}(-1)^{\varepsilon_{\alpha}}$, где $\bar{T}^{\alpha}$ представляют собой линейные комбинации связей $T_{\alpha}$, дуальные к $T_{\alpha}, \bar{T}^{\alpha} T_{\alpha}=0$.

Аналогично ситуации с оператором $\Delta$, если производящие уравнения допускают решение для $\bar{\Delta}$, линейное по $C^{A}$, то для любых величин, зависящих только от $\Pi_{*}^{\alpha}, \Pi_{\alpha}$, $\bar{\Delta}$-антискобка удовлетворяет сильному тож деству Якоби.

Однако согласно нашему предписанию аналог соотношений (4.49)-(4.51) теперь имеет вид

$$
\begin{aligned}
& \varepsilon\left(\Pi_{*}^{\alpha}\right)+\varepsilon\left(\Pi_{\alpha}\right)=1, \\
& \operatorname{gh}\left(\Pi_{*}^{\alpha}\right)+\operatorname{gh}\left(\Pi_{\alpha}\right)=1, \\
& \operatorname{deg}\left(\Pi_{*}^{\alpha}\right)+\operatorname{deg}\left(\Pi_{\alpha}\right)=0 .
\end{aligned}
$$

Мы видим, что знаки в правых частях соотношений (4.50) и (5.13) противоположны. Это означает, что импульсы $\Pi_{*}^{\alpha}$ и $\Pi_{\alpha}$, в отличие от $B^{\alpha}, B_{\alpha}^{*}$, ведут себя как "скрученные" поля и антиполя [8].

В дальнейшем мы подразумеваем, что решение производяших уравнений (4.28)-(4.31) и (5.1)-(5.3) действительно сушествует.

Коммутируя $\bar{\Delta}$ с третьим уравнением в $(4.28)$ и используя третье условие в $(4.29)$ и второе уравнение в (5.1), получаем

$$
\Sigma_{1}=(i \hbar)^{-1}\left[\Delta, S_{1}\right]
$$

где

$$
S_{1}=(i \hbar)^{-1}\left[\bar{\Delta}, \Sigma_{1}\right]+(i \hbar)^{-1}\left[\Delta, Y_{1}\right],
$$

$Y_{1}$ - произвольный фермионный оператор с $\operatorname{gh}\left(Y_{1}\right)=-1, \operatorname{deg}\left(Y_{1}\right)=1$. Для $S_{1}$ мы имеем

$$
\varepsilon\left(S_{1}\right)=0, \quad \operatorname{gh}\left(S_{1}\right)=0, \quad \operatorname{deg}\left(S_{1}\right)=1 .
$$

Подставляя представление (5.15) в первое из уравнений (4.28) и используя свойство (2.11), получаем

$$
\left[\Delta,\left(S_{1}, S_{1}\right)_{\Delta}\right]=0
$$


Коммутируя далее $\bar{\Delta}$ с уравнением (5.18), находим, аналогично (5.15),

$$
\left(S_{1}, S_{1}\right)_{\Delta}=i \hbar\left[\Delta, S_{2}\right]
$$

где

$$
S_{2}=\frac{1}{2}(i \hbar)^{-3}\left[\bar{\Delta},\left(S_{1}, S_{1}\right)_{\Delta}\right]+(i \hbar)^{-1}\left[\Delta, Y_{2}\right]
$$

$Y_{2}$ - произвольный фермионный оператор с $\operatorname{gh}\left(Y_{2}\right)=-1, \operatorname{deg}\left(Y_{2}\right)=2$. Для $S_{2}$ мы имеем

$$
\varepsilon\left(S_{2}\right)=0, \quad \operatorname{gh}\left(S_{2}\right)=0, \quad \operatorname{deg}\left(S_{2}\right)=2 .
$$

Рассмотрим теперь мастер-уравнение

$$
(S, S)_{\Delta}=i \hbar[\Delta, S]
$$

для бозонного оператора $S$ вида

$$
S=\sum_{k \geqslant 0} S_{k}, \quad S_{0}=G_{C B^{*}}, \quad \varepsilon\left(S_{k}\right)=0, \quad \operatorname{gh}\left(S_{k}\right)=0, \quad \operatorname{deg}\left(S_{k}\right)=k .
$$

Имеем

$$
\left[S_{0}, \Delta\right]=i \hbar \Delta, \quad\left[S_{0}, S_{k}\right]=-i \hbar k S_{k} .
$$

Подставляя разложение (5.23) в уравнение (5.22) и используя соотношения (5.24), мы получаем следующую цепочку уравнений:

$$
F_{k}=0, \quad k \geqslant 2,
$$

где

$$
\begin{aligned}
F_{k} & \equiv R_{k}-i \hbar(k-1)\left[\Delta, S_{k}\right], \\
R_{k} & \equiv \sum_{j=1}^{k-1}\left(S_{j}, S_{k-j}\right)_{\Delta}
\end{aligned}
$$

При $k=2$ уравнение (5.25) точно совпадает с (5.19), так что мы можем идентифицировать $S_{1}$ и $S_{2}$ в разложении (5.23) с соответствуюшими операторами в формулах (5.16) и (5.20). Используя тождество (2.10) для $Q=\Delta, B=S$, нетрудно вывести равенства

$$
\left[\Delta, R_{k}\right]=0
$$

при условии, что выполнены уравнения

$$
F_{m}=0, \quad m=2, \ldots, k-1 .
$$


Именно, из (2.10) следует

$$
6(S, F)_{\Delta}-[[S, F], \Delta]=4 i \hbar[\Delta, F]
$$

где

$$
F \equiv(S, S)_{\Delta}-i \hbar[\Delta, S]
$$

Предположим, что уравнения (5.29) выполняются. Рассматривая тождество (5.30) в секторе $\mathrm{c} \operatorname{deg}=k$, находим

$$
6\left(S_{0}, F_{k}\right)_{\Delta}-\left[\left[S_{0}, F_{k}\right], \Delta\right]=4 i \hbar\left[\Delta, F_{k}\right],
$$

откуда следует равенство

$$
(k-2)\left[\Delta, R_{k}\right]=0 .
$$

Наконец, коммутируя $\bar{\Delta}$ с уравнениями (5.28), мы получаем уравнение (5.25) для $m=k \mathrm{c}$

$$
\begin{gathered}
S_{k}=\frac{1}{k(k-1)}(i \hbar)^{-3}\left[\bar{\Delta}, R_{k}\right]+(i \hbar)^{-1}\left[\Delta, Y_{k}\right] \\
\varepsilon\left(Y_{k}\right)=1, \quad \operatorname{gh}\left(Y_{k}\right)=-1, \quad \operatorname{deg}\left(Y_{k}\right)=k .
\end{gathered}
$$

Таким образом, мы показали, что все операторы $S_{k}$ в разложении (5.23) сушествуют. Тем самым установлено, что мастер-уравнение (5.22) имеет решение, генерируемое оператором $\Sigma_{1}$ посредством соотношений (5.15), (5.19). Это решение описывает антискобочную алгебру, генерируемую БРСТ-инвариантными связями.

Рассмотрим простейший случай теории ранга 1 с алгеброй лиевского типа с постоянными структурными коэффициентами. Выбирая $C \overline{\mathcal{P}}$-упорядочение в гостовском секторе, мы имеем следующий БРСТ-оператор $\Omega$ :

$$
\Omega=C^{\alpha} T_{\alpha}+\frac{1}{2} C^{\beta} C^{\alpha} U_{\alpha \beta}^{\gamma} \overline{\mathcal{P}}_{\gamma}(-1)^{\varepsilon_{\beta}+\varepsilon_{\gamma}},
$$

с постоянными $U_{\alpha \beta}^{\gamma}$. Выберем простейшую возможную форму оператора $\Delta$ :

$$
\Delta=\Omega+\Pi_{*}^{\alpha} \Pi_{\alpha}(-1)^{\varepsilon_{\alpha}+1},
$$

так что соответствующий резольвентный оператор $\bar{\Delta}$ есть

$$
\bar{\Delta}=\bar{\Omega}-B^{\alpha} B_{\alpha}^{*}
$$

Для оператора $\Delta$, выбранного в форме (5.37), уравнение (5.19) имеет решение вида

$$
\begin{gathered}
S_{1}=\overline{\mathcal{P}}_{\alpha} B^{\alpha}+\frac{1}{2} B^{\beta} B^{\alpha} U_{\alpha \beta}^{\gamma} B_{\gamma}^{*}(-1)^{\varepsilon_{\beta}}, \\
\left(S_{1}, S_{1}\right)_{\Delta}=0, \quad S_{2}=0,
\end{gathered}
$$


а нильпотентный оператор $\Sigma_{1}$ в равенстве (5.15) дается формулой

$$
\begin{aligned}
\Sigma_{1}= & (i \hbar)^{-1}\left[\Delta, S_{1}\right]=B^{\alpha} \mathcal{T}_{\alpha}+\frac{1}{2} B^{\beta} B^{\alpha} U_{\alpha \beta}^{\gamma} \Pi_{\gamma}(-1)^{\varepsilon_{\beta}+\varepsilon_{\gamma}}+ \\
& +\Pi_{*}^{\alpha} \overline{\mathcal{P}}_{\alpha}(-1)^{\varepsilon_{\alpha}}-\Pi_{*}^{\beta} B^{\alpha} U_{\alpha \beta}^{\gamma} B_{\gamma}^{*}(-1)^{\varepsilon_{\beta}}
\end{aligned}
$$

где $\mathcal{T}_{\alpha}$ есть $C \overline{\mathcal{P}}$-упорядоченные БРСТ-инвариантные связи,

$$
\mathcal{T}_{\alpha}=(i \hbar)^{-1}\left[\Omega, \overline{\mathcal{P}}_{\alpha}\right]=T_{\alpha}+C^{\beta} U_{\alpha \beta}^{\gamma} \overline{\mathcal{P}}_{\gamma}(-1)^{\varepsilon_{\alpha}+\varepsilon_{\gamma}}
$$

В общем случае можно показать, что появление ненулевых $S_{k}, k \geqslant 2$, в разложении (5.23) является следствием антиканонического преобразования (2.14)-(2.16), примененного к оператору $S_{1}$, удовлетворяюшему однородному мастер-уравнению (5.40). Условно можно сказать, что правая часть уравнения (5.19) возникает из деформационного члена (2.19).

Отметим также, что решение вида (5.39), (5.40) остается справедливым и в случае, когда структурные коэффициенты $U_{\alpha \beta}^{\gamma}$ в (5.36) не постоянны, но удовлетворяют квазигрупповым условиям

$$
\left[U_{\alpha \beta}^{\gamma}, U_{\mu \nu}^{\rho}\right]=0, \quad\left[\left[T_{\alpha}, U_{\beta \gamma}^{\delta}\right], U_{\mu \nu}^{\rho}\right]=0
$$

\section{6. БРСТ-ИНВАРИАНТНАЯ АЛГЕБРА СВЯЗЕЙ В ТЕОРИЯХ РАНГА 1}

Здесь мы приведем явные формулы, которые могут оказаться полезными для применений в теориях ранга 1. Мы рассмотрим БРСТ-инвариантную алгебру в коммутаторной и антискобочной формах для наиболее популярных случаев вейлевского и виковского упорядочений гостовского сектора.

6.1. Вейлевски упорядоченный гостовский сектор. В случае вейлевски упорядоченного гостовского сектора теория ранга 1 описывается следующим БРСТ-оператором, линейным по гостовскому импульсу [9]:

$$
\begin{aligned}
\Omega= & C^{\alpha} T_{\alpha}+\frac{1}{6} C^{\beta} C^{\alpha} U_{\alpha \beta}^{\gamma} \overline{\mathcal{P}}_{\gamma}(-1)^{\varepsilon_{\beta}+\varepsilon_{\gamma}}+\frac{1}{6} C^{\alpha} U_{\alpha \beta}^{\gamma} \overline{\mathcal{P}}_{\gamma} C^{\beta}(-1)^{\varepsilon_{\beta}+\varepsilon_{\gamma}}+ \\
& +\frac{1}{6} \overline{\mathcal{P}}_{\gamma} U_{\alpha \beta}^{\gamma} C^{\beta} C^{\alpha}(-1)^{\varepsilon_{\beta}+\varepsilon_{\gamma}+\left(\varepsilon_{\alpha}+\varepsilon_{\beta}\right)\left(\varepsilon_{\gamma}+1\right)} .
\end{aligned}
$$

Исходная алгебра связей задается соотношениями “инволюции” [9]

$$
\left[T_{\alpha}, T_{\beta}\right]=\frac{i \hbar}{2}\left(U_{\alpha \beta}^{\gamma} T_{\gamma}+T_{\gamma} U_{\alpha \beta}^{\gamma}(-1)^{\left(\varepsilon_{\alpha}+\varepsilon_{\beta}+1\right) \varepsilon_{\gamma}}\right)+\left(\frac{i \hbar}{2}\right)^{2}\left[U_{\alpha \delta}^{\gamma}, U_{\gamma \beta}^{\delta}\right](-1)^{\varepsilon_{\delta}\left(\varepsilon_{\beta}+1\right)}
$$


БРСТ-инвариантные связи равны

$$
\begin{aligned}
\mathcal{T}_{\alpha} & =(i \hbar)^{-1}\left[\Omega, \overline{\mathcal{P}}_{\alpha}\right]= \\
& =T_{\alpha}+\frac{1}{2}\left(C^{\beta} U_{\beta \alpha}^{\gamma} \overline{\mathcal{P}}_{\gamma}(-1)^{\varepsilon_{\alpha}+\varepsilon_{\gamma}}+\overline{\mathcal{P}}_{\gamma} U_{\alpha \beta}^{\gamma} C^{\beta}(-1)^{\varepsilon_{\alpha}+\left(\varepsilon_{\alpha}+\varepsilon_{\beta}+1\right) \varepsilon_{\gamma}}\right) .
\end{aligned}
$$

Их коммутаторная алгебра есть

$$
\begin{aligned}
& {\left[\mathcal{T}_{\alpha}, \mathcal{T}_{\beta}\right]=} \frac{i \hbar}{2}\left(U_{\alpha \beta}^{\gamma} \mathcal{T}_{\gamma}+\mathcal{T}_{\gamma} U_{\alpha \beta}^{\gamma}(-1)^{\left(\varepsilon_{\alpha}+\varepsilon_{\beta}+1\right) \varepsilon_{\gamma}}\right)+ \\
&+\frac{1}{2}\left(\left[\Omega, U_{\alpha \beta}^{\gamma}\right] \overline{\mathcal{P}}_{\gamma}(-1)^{\varepsilon_{\alpha}+\varepsilon_{\beta}+\varepsilon_{\gamma}}-\overline{\mathcal{P}}_{\gamma}\left[\Omega, U_{\alpha \beta}^{\gamma}\right](-1)^{\left(\varepsilon_{\alpha}+\varepsilon_{\beta}\right) \varepsilon_{\gamma}}\right) \\
& {\left[\overline{\mathcal{P}}_{\alpha}, \overline{\mathcal{P}}_{\beta}\right]=0, \quad\left[\overline{\mathcal{P}}_{\alpha}, \mathcal{T}_{\beta}\right]=i \hbar U_{\alpha \beta}^{\gamma} \overline{\mathcal{P}}_{\gamma}(-1)^{\varepsilon_{\alpha}+\varepsilon_{\beta}+\varepsilon_{\gamma}} }
\end{aligned}
$$

Видно, что расширение, генерируемое третьим членом в правой части соотношения (6.2), отсутствует в формуле (6.4), а вместо этого в (6.4) присутствует примесь гостовских импульсов $\overline{\mathcal{P}}_{\alpha}$.

Антискобочная алгебра, соответствующая коммутаторной алгебре (6.4), (6.5), есть

$$
\begin{gathered}
\left(\mathcal{T}_{\alpha}, \mathcal{T}_{\beta}\right)_{\Omega}=0, \quad\left(\overline{\mathcal{P}}_{\alpha}, \overline{\mathcal{P}}_{\beta}\right)_{\Omega}=(i \hbar)^{2} U_{\alpha \beta}^{\gamma} \overline{\mathcal{P}}_{\gamma}(-1)^{\varepsilon_{\alpha}+\varepsilon_{\beta}+\varepsilon_{\gamma}} \\
\left(\overline{\mathcal{P}}_{\alpha}, \mathcal{T}_{\beta}\right)_{\Omega}=\frac{1}{2} i \hbar\left[\mathcal{T}_{\alpha}, \mathcal{T}_{\beta}\right](-1)^{\varepsilon_{\alpha}}
\end{gathered}
$$

6.2. Виковски упорядоченный гостовский сектор. Как обычно, виковский гостовский сектор описьвается двумя наборами виковских пар, $\left(C^{\alpha}, \bar{C}_{\alpha}^{\dagger}\right)$ и $\left(\bar{C}_{\alpha}, C^{\dagger \alpha}\right)$, с ненулевыми коммутаторами

$$
\left[C^{\alpha}, \bar{C}_{\beta}^{\dagger}\right]=\delta_{\beta}^{\alpha}, \quad\left[\bar{C}_{\alpha}, C^{\dagger \beta}\right]=\delta_{\alpha}^{\beta} .
$$

В теории ранга 1 виковски упорядоченный БРСТ-оператор равен [9]

$$
\begin{aligned}
\Omega= & T_{\alpha}^{\dagger} C^{\alpha}+C^{\dagger \alpha} T_{\alpha}+\left(\frac{1}{2} \bar{C}_{\gamma}^{\dagger} U_{\alpha \beta}^{\dagger \gamma} C^{\alpha} C^{\beta}+\frac{1}{2} C^{\dagger \beta} C^{\dagger \alpha} U_{\alpha \beta}^{\gamma} \bar{C}_{\gamma}+\right. \\
& \left.+C^{\dagger \alpha} \bar{U}_{\alpha \beta}^{\gamma} \bar{C}_{\gamma} C^{\beta}+C^{\dagger \beta} \bar{C}_{\gamma}^{\dagger} \bar{U}_{\alpha \beta}^{\dagger \gamma} C^{\alpha}\right)(-1)^{\varepsilon_{\beta}} .
\end{aligned}
$$

Исходная алгебра связей задается соотношениями “инволюции” [9]

$$
\begin{gathered}
{\left[T_{\alpha}, T_{\beta}\right]=U_{\alpha \beta}^{\gamma} T_{\gamma}, \quad\left[T_{\beta}^{\dagger}, T_{\alpha}^{\dagger}\right]=T_{\gamma}^{\dagger} U_{\alpha \beta}^{\dagger \gamma},} \\
{\left[T_{\alpha}, T_{\beta}^{\dagger}\right]=\bar{U}_{\alpha \beta}^{\gamma} T_{\gamma}+T_{\gamma}^{\dagger} \bar{U}_{\beta \alpha}^{\dagger \gamma}+\bar{U}_{\alpha \delta}^{\gamma} \bar{U}_{\beta \gamma}^{\dagger \delta}(-1)^{\varepsilon_{\gamma} \varepsilon_{\delta}} .}
\end{gathered}
$$

БРСТ-инвариантные связи равны

$$
\begin{aligned}
& \mathcal{T}_{\alpha}=\left[\bar{C}_{\alpha}, \Omega\right]=T_{\alpha}+C^{\dagger \beta} U_{\beta \alpha}^{\gamma} \bar{C}_{\gamma}(-1)^{\varepsilon_{\alpha}}+\bar{U}_{\alpha \beta}^{\gamma} \bar{C}_{\gamma} C^{\beta}(-1)^{\varepsilon_{\beta}}+\bar{C}_{\gamma}^{\dagger} \bar{U}_{\beta \alpha}^{\dagger \gamma} C^{\beta}(-1)^{\varepsilon_{\alpha}}, \\
& \mathcal{T}_{\alpha}^{\dagger}=\left[\Omega, \bar{C}_{\alpha}^{\dagger}\right]=T_{\alpha}^{\dagger}+\bar{C}_{\gamma}^{\dagger} U_{\beta \alpha}^{\dagger \gamma} C^{\beta}(-1)^{\varepsilon_{\alpha}}+C^{\dagger \beta} \bar{C}_{\gamma}^{\dagger} \bar{U}_{\alpha \beta}^{\dagger \gamma}(-1)^{\varepsilon_{\beta}}+C^{\dagger \beta} \bar{U}_{\beta \alpha}^{\gamma} \bar{C}_{\gamma}(-1)^{\varepsilon_{\alpha}}
\end{aligned}
$$


Ненулевые соотношения их коммутаторной алгебры суть

$$
\begin{gathered}
{\left[\mathcal{T}_{\alpha}, \mathcal{T}_{\beta}\right]=U_{\alpha \beta}^{\gamma} \mathcal{T}_{\gamma}+\left[\Omega, U_{\alpha \beta}^{\gamma}\right] \bar{C}_{\gamma}(-1)^{\varepsilon_{\alpha}+\varepsilon_{\beta}},} \\
{\left[\mathcal{T}_{\beta}^{\dagger}, \mathcal{T}_{\alpha}^{\dagger}\right]=\mathcal{T}_{\gamma}^{\dagger} U_{\alpha \beta}^{\dagger \gamma}+\bar{C}_{\gamma}^{\dagger}\left[U_{\alpha \beta}^{\dagger \gamma}, \Omega\right](-1)^{\varepsilon_{\alpha}+\varepsilon_{\beta}},} \\
{\left[\mathcal{T}_{\alpha}, \mathcal{T}_{\beta}^{\dagger}\right]=\bar{U}_{\alpha \beta}^{\gamma} \mathcal{T}_{\gamma}+\mathcal{T}_{\gamma}^{\dagger} \bar{U}_{\beta \alpha}^{\dagger \gamma}+\left[\Omega, \bar{U}_{\alpha \beta}^{\gamma}\right] \bar{C}_{\gamma}(-1)^{\varepsilon_{\alpha}+\varepsilon_{\beta}}+} \\
\left.\quad+\bar{C}_{\gamma}^{\dagger} \bar{U}_{\beta \alpha}^{\dagger \gamma}, \Omega\right](-1)^{\varepsilon_{\alpha}+\varepsilon_{\beta}}, \\
{\left[\bar{C}_{\alpha}, \mathcal{T}_{\beta}\right]=U_{\alpha \beta}^{\gamma} \bar{C}_{\gamma}(-1)^{\varepsilon_{\beta}}, \quad\left[\mathcal{T}_{\beta}^{\dagger}, \bar{C}_{\alpha}^{\dagger}\right]=\bar{C}_{\gamma}^{\dagger} U_{\alpha \beta}^{\dagger \gamma}(-1)^{\varepsilon_{\beta}},} \\
{\left[\mathcal{T}_{\alpha}, \bar{C}_{\beta}^{\dagger}\right]=\bar{U}_{\alpha \beta}^{\gamma} \bar{C}_{\gamma}(-1)^{\varepsilon_{\beta}}+\bar{C}_{\gamma}^{\dagger} \bar{U}_{\beta \alpha}^{\dagger \gamma}(-1)^{\varepsilon_{\alpha}},} \\
{\left[\bar{C}_{\beta}, \mathcal{T}_{\alpha}^{\dagger}\right]=\bar{C}_{\gamma}^{\dagger} \bar{U}_{\alpha \beta}^{\dagger \gamma}(-1)^{\varepsilon_{\beta}}+\bar{U}_{\beta \alpha}^{\gamma} \bar{C}_{\gamma}(-1)^{\varepsilon_{\alpha}} .}
\end{gathered}
$$

Как и в случае вейлевски упорядоченного гостовского сектора, видно, что расширение, генерируемое третьим членом в правой части соотношения (6.11), отсутствует в формуле (6.14), а вместо этого в (6.14) присутствует примесь гостовских импульсов $\bar{C}_{\alpha}$ и $\bar{C}_{\alpha}^{\dagger}$.

Антискобочная алгебра, соответствующая коммутаторной алгебре (6.12)-(6.17), есть

$$
\begin{gathered}
\left(\mathcal{T}_{\alpha}, \mathcal{T}_{\beta}\right)_{\Omega}=0, \quad\left(\mathcal{T}_{\alpha}^{\dagger}, \mathcal{T}_{\beta}^{\dagger}\right)_{\Omega}=0, \quad\left(\mathcal{T}_{\alpha}, \mathcal{T}_{\beta}^{\dagger}\right)_{\Omega}=0, \\
\left(\bar{C}_{\alpha}, \bar{C}_{\beta}\right)_{\Omega}=U_{\alpha \beta}^{\gamma} \bar{C}_{\gamma}, \quad\left(\bar{C}_{\beta}^{\dagger}, \bar{C}_{\alpha}^{\dagger}\right)_{\Omega}=\bar{C}_{\gamma}^{\dagger} U_{\alpha \beta}^{\dagger \gamma}, \\
\left(\bar{C}_{\alpha}, \bar{C}_{\beta}^{\dagger}\right)_{\Omega}=\bar{U}_{\alpha \beta}^{\gamma} \bar{C}_{\gamma}(-1)^{\varepsilon_{\beta}}+\bar{C}_{\gamma}^{\dagger} \bar{U}_{\beta \alpha}^{\dagger \gamma}(-1)^{\varepsilon}, \\
\left(\bar{C}_{\alpha}, \mathcal{T}_{\beta}\right)_{\Omega}=\frac{1}{2}\left[\mathcal{T}_{\alpha}, \mathcal{T}_{\beta}\right], \quad\left(\mathcal{T}_{\beta}^{\dagger}, \bar{C}_{\alpha}^{\dagger}\right)_{\Omega}=\frac{1}{2}\left[\mathcal{T}_{\beta}^{\dagger}, \mathcal{T}_{\alpha}^{\dagger}\right], \\
\left(\bar{C}_{\alpha}, \mathcal{T}_{\beta}^{\dagger}\right)_{\Omega}=\frac{1}{2}\left[\mathcal{T}_{\alpha}, \mathcal{T}_{\beta}^{\dagger}\right], \quad\left(\mathcal{T}_{\beta}, \bar{C}_{\alpha}^{\dagger}\right)_{\Omega}=\frac{1}{2}\left[\mathcal{T}_{\beta}, \mathcal{T}_{\alpha}^{\dagger}\right] .
\end{gathered}
$$

Явные формулы, приведенные в п. 6.1 и 6.2 , наглядно демонстрируют существование очевидного дуализма между двумя альтернативными формами БРСТ-инвариантной алгебры связей, описываемого обшим соответствием

$$
\mathcal{T}, \overline{\mathcal{P}},[\cdot, \cdot] \quad \Longleftrightarrow \overline{\mathcal{P}}, \mathcal{T},(\cdot, \cdot)_{\Omega} .
$$

\section{7. ЗАКЛЮЧЕНИЕ}

Таким образом, сформулирован новый подход к квантованию калибровочно-инвариантных динамических систем, который, по сушеству, основан на концепции БРСТ-инвариантных связей. В основе всей конструкции лежит новый нильпотентный "БРСТзаряд" $\Sigma_{1}$, который определен в дополнительно расширенном фазовом пространстве. Прежнее расширенное фазовое пространство, координатами которого являются исходные фазовые переменные и обычные госты, теперь становится новым "исходным" пространством. Новые канонические пары $\left(C^{A}, \overline{\mathcal{P}}_{A}\right)(4.23),(4.10)$ играют роль новых "минимальных" гостов, в то время как новое квантовое число, степень (deg), играет роль нового гостовского числа. Рассматривая эти новые канонические пары как "минимальные" госты, мы можем ввести новые антигосты $\left(\mathcal{P}^{A}, \bar{C}_{A}\right), \varepsilon\left(\mathcal{P}^{A}\right)=\varepsilon\left(\bar{C}_{A}\right)=\varepsilon_{A}+1$, 
$\operatorname{gh}\left(\mathcal{P}^{A}\right)=-\operatorname{gh}\left(\bar{C}_{A}\right)=\{1 ; 2\}, \operatorname{deg}\left(\mathcal{P}^{A}\right)=-\operatorname{deg}\left(\bar{C}_{A}\right)=\{1 ; 1\}$, и лагранжевы множители $\left(\lambda^{A}, \pi_{A}\right), \varepsilon\left(\lambda^{A}\right)=\varepsilon\left(\pi_{A}\right)=\varepsilon_{A}, \operatorname{gh}\left(\lambda^{A}\right)=-\operatorname{gh}\left(\pi_{A}\right)=\{0 ; 1\}, \operatorname{deg}\left(\lambda^{A}\right)=-\operatorname{deg}\left(\pi_{A}\right)=$ $\{0 ; 0\}$, с ненулевыми коммутаторами

$$
\left[\mathcal{P}^{A}, \bar{C}_{B}\right]=i \hbar \delta_{B}^{A}, \quad\left[\lambda^{A}, \pi_{B}\right]=i \hbar \delta_{B}^{A}
$$

и затем обычным образом построить новый клибровочно-фиксированный унитаризующий гамильтониан.

Чтобы реализовать эту программу, мы должны сначала построить "минимальный" гамильтониан $\Xi$, который удовлетворяет уравнениям

$$
\begin{gathered}
{\left[\Sigma_{1}, \Xi\right]=0,} \\
\varepsilon(\Xi)=0, \quad \operatorname{gh}(\Xi)=0, \quad \operatorname{deg}(\Xi)=0,
\end{gathered}
$$

и граничному условию

$$
\Xi=\mathcal{H}+\cdots, \quad[\mathcal{H}, \Omega]=0 .
$$

Затем мы построим полный унитаризуюший гамильтониан в стандартной форме,

$$
\begin{gathered}
H=\Xi+(i \hbar)^{-1}[\Sigma, \Psi], \\
\varepsilon(\Psi)=1, \quad \operatorname{gh}(\Psi)=-1, \quad \operatorname{deg}(\Psi)=-1,
\end{gathered}
$$

где

$$
\Sigma=\Sigma_{1}+\pi_{A} \mathcal{P}^{A}, \quad \Psi=\bar{C}_{A} \chi^{A}+\overline{\mathcal{P}}_{A} \lambda^{A}
$$

и $\chi^{A}$ есть оператор, фиксируюший калибровку. Исходный гамильтониан и связи первого рода содержатся в $\mathcal{H}$ и $\Omega$, соответственно, в членах низшего порядка разложения в ряд по степеням операторов обычных гостов $\left(C^{\alpha}, \overline{\mathcal{P}}_{\alpha}\right)$.

Физические наблюдаемые коммутируют с $\Sigma$, а физические состояния аннигилируются этим оператором. Если подходяшим образом определить физическое скалярное произведение, то физические матричные элементы физических операторов не будут зависеть от калибровки. Тогда можно перейти к унитарному пределу, выбирая унитарную калибровку вида

$$
\chi^{A}=0, \quad \chi^{A} \equiv\left\{\chi^{\alpha} ; C^{\alpha}\right\},
$$

где $\chi^{\alpha}$ - обычная калибровка по отношению к исходным связям $T_{\alpha}$, и установить совпадение физических амплитуд переходов ( $S$-матришы) с физическими амплитудами переходов в стандартном БРСТ-БВФ-подходе. Однако при использовании обших релятивистских калибровок расширенный формализм сушественно обобщает стандартный, сохраняя более явной БРСТ-симметрию производящего механизма калибровочной алгебры.

В заключение сделаем следуюшее замечание. Рассмотрим стандартную форму [2] унитаризуюшего гамильтониана в БРСТ-БВ

$$
H=\mathcal{H}+(i \hbar)^{-1}[Q, \Psi]
$$


где $\mathcal{H}$ есть минимальный гамильтониан,

$$
\begin{gathered}
Q=\Omega+\pi_{\alpha} \mathcal{P}^{\alpha}, \quad \Psi=\bar{C}_{\alpha} \chi^{\alpha}+\overline{\mathcal{P}}_{\alpha} \lambda^{\alpha} \\
{[\Omega, \Omega]=0, \quad[\mathcal{H}, \Omega]=0}
\end{gathered}
$$

$\Omega$ - минимальный БРСТ-оператор, $\Psi$ - калибровочный фермион. Мы имеем

$$
H=\mathcal{H}+(i \hbar)^{-1}\left[\Omega, \overline{\mathcal{P}}_{\alpha}\right] \lambda^{\alpha}+\overline{\mathcal{P}}_{\alpha} \mathcal{P}^{\alpha}+\pi_{\alpha} \chi^{\alpha}+\bar{C}_{\alpha}(i \hbar)^{-1}\left[\chi^{\alpha}, \Omega\right]
$$

Во втором и третьем членах в правой части мы узнаем связи $\mathcal{T}_{\alpha}$ и $X_{\alpha}$ в их простейшей возможной форме,

$$
\mathcal{T}_{\alpha}=(i \hbar)^{-1}\left[\Omega, \overline{\mathcal{P}}_{\alpha}\right], \quad-X_{\alpha}=\overline{\mathcal{P}}_{\alpha}
$$

с $\lambda^{\alpha}$ и $-\mathcal{P}^{\alpha}$ в качестве соответствующих лагранжевых множителей. Четвертый и пятьй члены фиксируют калибровку, причем $\chi^{\alpha}$ и $(i \hbar)^{-1}\left[\chi^{\alpha}, \Omega\right]$ являются операторами, фиксируюшими калибровку $\mathcal{T}_{\alpha}$ и $X_{\alpha}$, соответственно, а $\pi_{\alpha}, \bar{C}_{\alpha}$ - соответствуюшие лагранжевы множители.

Таким образом, выясняется, что фактически стандартный гамильтониан (7.9), (7.10) построен именно в терминах “стандартных" БРСТ-инвариантных связей (7.13) и соответствуюших фиксируюших калибровку операторов. Однако по сравнению с операторами $T_{A}$ в общем положении, которые удовлетворяют только соотношениям (4.34), (4.37), связи (7.13) являются довольно специальными в том смысле, что рассматриваемые как объекты линейного комбинирования, они соответствуют выбору специального базиса.

В противоположность ситуации со стандартным гамильтонианом, новый гамильтониан (7.12), определенньй в дополнительно расширенном фазовом пространстве, построен прямо в терминах обших операторов $T_{A}$, подчиненных только условиям (4.34), (4.37). Таким образом, введение новых переменных $\left(C^{A}, \overline{\mathcal{P}}_{A}\right), \quad\left(\mathcal{P}^{A}, \bar{C}_{A}\right)$, $\left(\lambda^{A}, \pi_{A}\right)$ есть цена возможности произвольно выбирать базис БРСТ-инвариантных связей $T_{A}$ общего положения.

Наконец, следует отметить, что переход от формализма, описываемого формулами (7.9)-(7.11), к формализму, описываемому формулами (7.2)-(7.7), представляется только первым шагом в, возможно бесконечной, иерархии гамильтонианов.

Благодарности. Авторы признательны М. Григорьеву и А. Семихатову за плодотворные дискуссии. И. А. Баталин благодарен Р. Марнелиусу за важные обсуждения аспектов, связанных с квантовыми антискобками и операторнозначным мастер-уравнением. Работа И. А. Баталина частично поддержана Президентским грантом № 00-15-96566, грантом РФФИ № 02-01-00930 и грантом INTAS № 00-00262. Работа И. В. Тютина частично поддержана Президентским грантом № 00-15-96566, грантом РФФИ № 02-02-16944 и грантом INTAS № 00-00262. 


\section{Список литературы}

[1] C. Becchi, A. Rouet, R. Stora. Phys. Lett. B. 1974. V. 54. P. 344; Commun. Math. Phys. 1975. V. 42. P. 127; Ann. Phys. 1976. V. 98. Р. 287; И. В. Тютин. Калибровочная инвариантность в теории поля и статистической физике в операторной формулировке. Препринт ФИАН № 39. М.: ФИАН, 1975; E. S. Fradkin, G. A. Vilkovisky. Phys. Lett. B. 1975. V. 55. P. 224; Quantization of relativistic systems with constraints: equivalence of canonical and covariant formalisms in quantum theory of gravitational field. CERN preprint TH 2332-CERN. Geneva: CERN, 1977.

[2] I. A. Batalin, G. A. Vilkovisky. Phys. Lett. B. 1977. V. 69. P. 309; E. S. Fradkin, T. E. Fradkina. Phys. Lett. B. 1978. V. 72. P. 343.

[3] I. A. Batalin, E. S. Fradkin. Phys. Lett. B. 1983. V. 128. P. 303; Riv. Nuovo Cimento. 1986. V. 9. P. 1; T. Kugo, I. Ojima. Progr. Theor. Phys. Suppl. 1979. V. 66. P. 1; K. Nishijima. Nucl. Phys. B. 1984. V. 238. P. 601; Progr. Theor. Phys. 1988. V. 80. P. 897; P. 905; M. Henneaux. Phys. Rep. 1985. V. 126. P. 1; M. Henneaux, C. Teitelboim. Quantization of Gauge Sistems. Princeton: Princeton Univ. Press, 1992.

[4] M. B. Green, J. H. Shwarz, E. Witten. Superstring Theory. V. 1. Cambridge: Cambridge Univ. Press, 1987.

[5] I. A. Batalin, R. Marnelius. Phys. Lett. B. 1998. V. 441. P. 243; hep-th/9806114; Nucl. Phys. B. 1998. V. 551. P. 450; hep-th/9809208; Mod. Phys. Lett. A. 1999. V. 14. P. 1643; hep-th/9905193; Int. J. Mod. Phys. A. 2000. V. 15. P. 2077; hep-th/9909223.

[6] I. A. Batalin, R. Marnelius. Phys. Lett. B. 1998. V. 434. P. 312; hep-th/9805084.

[7] И. А. Баталин, Р. Марнелиус. ТМФ. 1999. Т. 120. С. 358; hep-th/9905083; I. A. Batalin, R. Marnelius. Int. J. Mod. Phys. A. 1999. V. 14. P. 5049; hep-th/9909210.

[8] A.S. Cattaneo, G. Felder. Commun. Math. Phys. 2000. V. 212. P. 591; math.QA/9902090; Mod. Phys. Lett. A. 2001. V. 16. P. 179; hep-th/0102208; I. A. Batalin, R. Marnelius. Phys. Lett. B. 2001. V. 512. P. 225; hep-th/0105190; Superfield algorithms for topological field theories. In: Multiple Facets of Quantization and Supersymmetry: Memorial Volume Dedicated to M. Marinov. Ed. M. Olshanetsky, A. Vainshtein. River Edge: World Scientific, 2002. P. 233; hep-th/0110140.

[9] I. A. Batalin, E. S. Fradkin. Ann. Inst. Henri Poincare. 1988. V. 49. P. 145.

Поступила в редакцию 17.III.2003 г. 